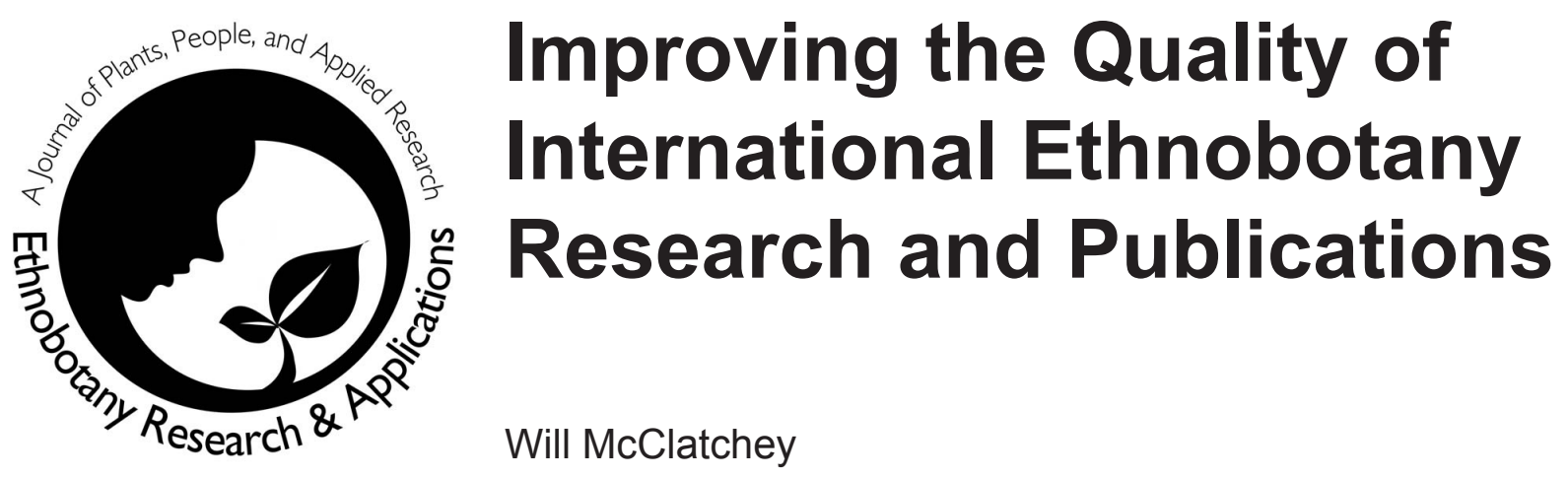

\title{
Editorial
}

I am tired of sending rejection letters to authors. It is particularly troublesome because the same kinds of problems seem to plague many authors.

It is interesting that there is a clear pattern of problems that cause ethnobotany manuscripts to be rejected by reviewers and editors of Ethnobotany Research and Applications. Comments from editors of related journals lead to the conclusion that similar problems abound. Appendix 1 illustrates the kinds of problems encountered.

First, the problems should be divided into two categories: Poor scientific-style presentation and poor quality science.

A poor quality of the presentation of the science indicates that the author lacks something: writing skills, fluency in the language being used, concern about quality, positive models of quality writing, or a variety of related attributes. There are many different writing styles and good authors learn what works for them and what does not. For the rest of us, template formats are useful for the organization of our work in order to provide clear presentations. Appendix 2 is a simple procedural template that if followed will overcome many structural problems seen in manuscripts rejected for publication. When combined with the advice in Appendix 1, this should produce a good publication if the research itself was conducted well.

Poor quality science is usually noted after the work has been completed and it is too late for corrections. It would be easy to simply reject work like this out of hand, except that the pattern that is seen indicates a deeper issue that needs to be addressed. I see the real problem behind poor quality ethnobotany research, particularly from scholars based in the United Nations Least Developing Countries, Landlocked Developing Countries, and Small Island Developing States (un.org/special-rep/ohrlls/

ohrlls/) as related to limited access to information on basic research methods.

An example of a typical conversation with an author of a manuscript with many of the problems in Appendix 1 may be insightful. Appendix 3 is a contrived conversation that includes elements of conversations that I have had with real authors. The key point of the conversation is to point out that the discrepancies in the quality of scientific research may be due to the inequitable access to current research and more specifically, current research methods.

\section{Standards of Research Practice}

Ethnobotany researchers are expected to conduct research that is original, reproducible, and which results in new understandings of human interactions with plants. The work may be intended to address theoretical or applied questions, but it should address questions. The colonial era days of producing lists of useful plants that only addresses the question "what is out there that is valu-

\section{Correspondence}

Will C. McClatchey, Department of Pharmaceutical Botany and Pharmacognosy, Faculty of Pharmacy, Khon Kaen University, Khon Kaen, 40002. THAILAND

mcclatch@hawaii.edu

Ethnobotany Research \& Applications 4:001-009 (2006) 
able?" should be behind us. The same standards should be held for researchers from any part of the world. However, unequal access to training, literature, and interactions with other scholars, often leave those from developing countries (or even minority communities in developed countries) in a difficult position when it comes to conducting research and subsequently getting it published in peer-reviewed journals.

\section{A Proposal to Remedy the Lack of Access to Ethnobotany Research Literature}

In many countries, access to scientific journals and current books is limited or non-existent. Researchers are faced with the task of using older literature, often locally produced books, that portray the goal of ethnobotanical research as producing lists of information about uses of plants. This is often supplemented by a scattering of research articles that reference published theories, hypotheses, and methods, but which are unavailable to the researchers. In this situation, the researchers do what is natural: to follow the books of lists as models and attempt to use the methodological terms in the literature even if they are not clearly understood.

I propose a general solution (Table 1) to the problem of access to modern ethnobotany research methods. Hopefully, this solution will increase the overall quality of work being conducted in the world and will provide opportunities for authors with limited access to the literature to produce and publish clear papers that review the methods. This should be an opportunity that is particularly well suited for graduate students whom are often required to produce reviews of research methods but rarely are able to convert these directly into peer-reviewed publications.

In order to promote on-going methodological development, no topic will be considered as final or complete. Authors wishing to publish a new publication on the method that improves clarity and completeness will be encouraged to do so. A comment and feedback web page will be linked to each document in order to scholars to provide brief updates, comments, or links to additional related resources. Comments will be screened through the journal editors in order to avoid the common problems associated with message boards and open web-logs that become clogged with meaningless information and unsolicited advertisements.

A hypothesis that results from this brief editorial follows the classic strong inference logic of Platt (1964). I predict that as research methods become more readily available, the quality of research conducted will improve. This will partially be measured by rejection of fewer manuscripts for the reasons listed in Appendix 1 and by an increase in the number of manuscripts reviewed that use and cite the methods that will be published in these articles.

Table 1. A proposed general solution to provide equal access to modern ethnobotanical research methods using the Internet.

Goal: Ethnobotany Research and Applications will publish an extensive set of methodological review articles. Each article will be posted on the journal web site with the intention of free and easy Internet access for any person in the world. The articles will also be distributed via e-mail, $C D$, and in print to locations where they are needed where the Internet is not accessible.

Format: Each article will:

- Clearly describe a specific research method in a step-by-step fashion.

- Include example formats for data collection, procedures for the analysis of the data, and guidelines for the interpretation of the results.

- Discuss applications of the research method.

- Discuss ethical, legal and intellectual concerns.

- $\quad$ Provide examples of common problems and solutions with the method.

- Include a broad literature review, from a wide range of publications, illustrating where the method has been used and which discuss methodological variations.

- Provide examples of hypotheses which have been tested or theoretical questions addressed by the research method..

Call for Manuscripts: Scholars from around the world are encouraged to submit manuscripts for consideration that address the above points about a specific method. These will be peer-reviewed, and if accepted, published on-line in Ethnobotany Research and Applications at www.ethnobotanyjournal.org. Each article will be linked to a special Internet comment page where critiques, additions, changes, and other kinds of comments may be posted. Authors will be given the opportunity to reply to comments. Authors wishing to reserve a particular method may do so for a three month period of preparation by making a request to the editor at: editor@ethnobotanyjournal.org.

\section{References Cited}

Chicago Manual of Style. 1993. The Chicago Manual of Style, 14th revised edition. University of Chicago Press, Chicago.

press.uchicago.edu/Misc/Chicago/cmosfaq/about.html

Cunningham, A.B. 2001. Applied Ethnobotany: People, wild plant use and conservation. Earthscan Publications Ltd, London. 


\section{McClatchey - Improving the Quality of International Ethnobotany Research and Publications}

Gordon, R.G., Jr. 2005 onwards (continuously updated). Editor of Ethnologue: Languages of the World. Fifteenth edition. Summer Institute of Linguistics, International, Dallas, Texas. www.ethnologue.com

Holmgren, P.K. \& N.H. Holmgren. 1998 onwards (continuously updated). Index Herbariorum. New York Botanical Garden, Bronx. sciweb.nybg.org/science2/IndexHerbariorum.asp

International Society of Ethnobiology. n.d. Guidelines for research, collections, databases and publications. guallart.dac.uga.edu/guidelines

Martin, G.J. 1995. Ethnobotany: A methods manual. Cambridge University Press, Cambridge,

UK.

McClatchey, W. 2005. Exorcizing Misleading Terms from Ethnobotany. Ethnobotany Research and Applications $3: 1-4$

McClatchey, W. \& K. Winter. 2005. Publication of Ethnobotanical Research in Local Languages. Ethnobotany Research and Applications 3:279-282.

Platt, J.R. 1964. Strong Inference. Science 146:347-353.

Society for Economic Botany. 1995. Guidelines of Professional Ethics. econbot.org/ethics/professional_ethics.html

Stevens, P.F. 2001 onwards (continuously updated). Angiosperm Phylogeny Website. Version 6, May 2005. Missouri Botanical Garden, St. Louis. mobot.org/MOBOT/research/APweb/

The Angiosperm Phylogeny Group. 2003. An update of the Angiosperm Phylogeny Group classification for the orders and families of flowering plants: APG II. Botanical Journal of the Linnean Society 141:399-436. 
Appendix 1. Kinds of problems frequently encountered by reviewers of manuscripts. These result in rejection of the manuscript or lengthy delays in publication while problems are resolved. Obvious solutions to each problem are listed in [ ]. This may serve as a checklist for authors of future manuscripts submitted for consideration for publication in Ethnobotany Research and Applications.

1. Problems with scientific reproducibility:

- $\quad$ No hypothesis or research objective. [Most research should state a clear hypothesis that was tested and include a discussion of the analysis of the hypothesis in light of new data.]

- No methods presented or presentation is vague. [Clearly reproducible scientific methods must be used.]

- No results presented or results and discussion of results are mixed so that it is not clear what is a result of the current research. [Results that are reported must relate directly to the methods that were described. It must be clear that these results were obtained in this study and that they are not data from another study. The results presentation must be distinct from the evaluation (discussion) of the results.]

- Vague or incorrect use of statistics. [Statistical methods used must be appropriate and indicate significance.]

- Inappropriate use of literature. Often this involves citing references that are not needed or not citing references that are needed. [Literature citations must be appropriately used to abbreviate long discussions and to contextualize the research within the larger framework of science. Avoid an excessive use of citations.]

- No discussion of results or a "discussion" that is simply a restatement of results also illustrated in figures or tables. [Results MUST be analyzed and discussed in a thoughtful way. It is not sufficient to present a table of results with a paragraph or two loosely listed as discussion.]

- No conclusions about the research. [Conclusions should, when possible, contribute to theoretical understandings of plant - human interactions.]

\section{Problems with biological science:}

- Improper citation of biological organisms. [Biological organisms that are cited as Latin binominals must include the authority. If plants families are cited, those recognized by the Angiosperm Phylogeny Working Group (Stevens 2001, The Angiosperm Phylogeny Group 2003) should be used. This, and the related open access Tropicos database, are available on-line through the Missouri Botanical Garden web site: www.mobot.org.]

- $\quad$ Lack of physical evidence or proof of species being discussed. [Biological organisms that are cited as part of the results must include reference to voucher specimens prepared within the course of the research. Vouchers should be deposited in recognized international repositories such as herbaria. Index Herbariorum (Holmgren \& Holmgren 1998) is the on-line database of recognized herbaria from around the world and should be consulted.]

- Organisms and data about organisms are presented but it is unclear if or how the results are representative of living systems. [Biodiversity sampling methods must be explicit and evaluated for statistical significance.]

- Research is presented without a clear biological or geophysical context. [The biological context of the research must be clearly, but briefly, established as part of the introduction. The locations of the research must be able to be determined by the reader. Location names should be available in standard gazatters. Otherwise, maps or geographic coordinates must be provided.]

- Organisms are discussed without reference to their context (cultivated, etc.). [Lists of species used in locations must be evaluated and contextualized as part of a discussion.] 


\section{McClatchey - Improving the Quality of International Ethnobotany Research and Publications}

3. Problems with social science:

- Cultures or other groupings of people are named but it is unclear who is specifically being discussed. [Cultural groups/communities within which work is conducted must be clearly but briefly described. Ethnologue (Gordon 2005) includes general and specific cultural information.]

- Social settings and data about cultures are presented but it in unclear if or how the results are representative of cultural systems. [The social setting of research must be clearly established and the research results evaluated within this context.]

- The research results are clearly derived from information learned from people who use plants, but it is not clear that permission was granted to obtain or publish the information. [Informed consent must be used throughout the research process and should be clearly indicated in the manuscript. Each of the international ethnobotanical research societies (e.g., International Society of Ethnobiology n.d., Society for Economic Botany 1995) has developed a set of working guidelines for ethical research. These should be followed. There are also many Internet accessible sites discussing the informed consent process and research ethics.]

- $\quad$ No methods presented or presentation is vague. [Ethnographic and other social research methods must be explicitly but briefly described.]

- A list of plants and uses is presented but the context of the uses and a discussion of these uses is lacking. [Lists of uses of organisms in a location must be evaluated and contextualized as part of a discussion.]

- The context of the research and researchers is absent or vague. [The manuscript must clearly indicate who conducted the research, with whom it was conducted, the actual period over which the research took place, and where it was done. If the research locations are not already well known, or easily found in a readily available referenced publication, there should be a specially-constructed map that shows all of the relevant places.]

- Common plant names are presented but no information is provided about the source or verification of the names. [When vernacular names are used for organisms, these must be associated with voucher specimens that were positively identified by research participants (informants). Separate collections of voucher specimens or simply identifying species through dictionaries or lists of plant names is unacceptable.]

4. General problems:

- The manuscript is not written in acceptable language. [The manuscript must be written well in the language it is written in (all languages have rules and these should be followed). Grammatical errors will not be tolerated by editors and will not help reviewers to have a positive opinion of the manuscript. The Chicago Manual of Style (1993) is used for production of many English documents.]

- The manuscript is needlessly offensive to members of the community discussed or other cultures contrasted or compared with the community discussed. [Manuscripts should be written in ways that are respectful of cultures. Authors should avoid inflammatory language since it rarely supports scientific arguments. Authors should avoid using meaningless generic vocabulary and misleading terms (see McClatchey 2005).]

- The research is inappropriate for the journal. [Manuscripts that do not include any research results obtained from humans about their interactions with plants are not usually accepted for publication in Ethnobotany Research and Applications with the exception of theoretical papers about plant-human interactions. Researchers working on laboratory (only) analysis of plants, without any cultural context in the actual research, should submit elsewhere.] 
Appendix 2. Template outline of a clear paper discussing ethnobotanical research.

The first part of writing is NOT to write the title, abstract, key words, introduction or literature review. This is a common error that leads to many structural problems and gets the writing process off to a bad start. Presumably the researcher was up-to-date on the literature BEFORE conducting the research and used that opportunity to generate a meaningful and testable hypothesis and appropriate methods.

Part 1: Write about the research that you conducted:

- Begin by writing the hypothesis and/or related research objectives. Be as clear as possible. Prepare a list of references that have been read that point to the hypothesis or call for the research objectives to be obtained.

- Write about the methods that were used to test the hypothesis or examine the objective. Include only those methods that provided results used specifically in regards to the hypothesis or objective, not everything that you did as part of your study. When possible, reference the same methods used and described by others in order to minimize writing and to construct links to other research. If new methods were developed and used, then clearly describe the method in such a way that other researchers can follow the directions and produce comparable data. Prepare a list of references to research that used similar methods.

- Write about the methods used for ethical and statistical reasons. Be clear about why a method was used. When possible, reference arguments for using the methods as presented in other literature and do not repeat the arguments in this manuscript.

- Organize the results based upon the method used to generate them and do not mix results from different methods even if multiple methods were used to triangulate data.

- Produce tables, figures, ethnographic descriptions, etc. of the results. Statistical analyses and categorization (qualification) of results should be included. Do not include other interpretations of the results. [One of the worst problems for unskilled writers is to confuse results with the interpretation of results. Interpretation belongs in a discussion section.]

- Write a very simple results section that uses sentences to describe the links between the methods used and the tables, figures, ethnographic descriptions, etc. of results. In many cases, this is very short consisting of a few sentences or paragraphs.

- Write complete reference citations as they are used. It is easy to forget a reference along the way or loose track of the source of a quote or piece of information. Avoid abbreviations in this list and do not use et al. in the list even though it is used in the written text.

Part 2: Interpret the results in light of the hypothesis or objective and construct bridges between the interpretations of the results of the present research with past research and the larger context of the subject.

- Consider the ways that the results of the research relate to other research conducted in the past on related subjects. Make a list of the references of related research.

- Write clear discussions of the results. At first, discuss results in the order of their presentation in the results section (and indirectly the methods section). DO NOT REPEAT data from the results section in a redundant way but discuss it in light of the larger body of research in the world.

- There is a strong temptation to write introductory paragraphs explaining other research with discussions of theories or concepts advanced. Write these extra paragraphs with appropriate references and set them aside for the fourth part below.

- When writing the discussion, discuss theories and other research as if it has already been introduced earlier in the paper.

- Clearly and specifically evaluate hypotheses indicating if and how the research supports or rejects each hypothesis. 


\section{McClatchey - Improving the Quality of International Ethnobotany Research and Publications}

Part 3: Advance science through future research by producing logical theories and new hypotheses that are implied by the research conducted.

- Consider the theoretical implications of the research. When hypotheses are used with good methods, general theories of human interactions with plants will typically emerge and these should be clearly stated.

- Consider possible new ethical considerations raised by the research. Write about ethical problems faced and solutions used IF they have a direct impact on the quality of the work and/or serve as a useful critique of the methods employed.

- Do not introduce references to the literature in the conclusions. Rather, as it is clear that references to other literature are important, write referenced paragraphs that introduce the pertinent literature and set these aside for the fourth part below.

Part 4: Produce an introduction to past research that is appropriate to introduce this research and discuss the context of interpretation of the results. Avoid the strong temptation to write this part first. If you do, you are likely to include information that is not important. For instance, although the geographical location is important for most studies, the geology and details of the geographical history are generally inappropriate.

- Use the lists of references, topics, and extra paragraphs produced while writing the hypothesis, methods, discussion and conclusions to produce clear, concise, and accurately referenced paragraphs that introduce topics that are needed to contextualize the research.

- Write a brief biological description of the research site.

- Write a brief background of the culture or community including only information that is important to introduce the research topic.

- Organize the paragraphs in a logical order then write an introduction paragraph that provides leads to the topics discussed in this introduction.

- Write a bridging paragraph at the end of the introduction that makes it clear how the researcher developed the research hypothesis or objectives.

Part 5: The final part is to produce the remaining parts of the paper, including the abstract, title, keywords, acknowledgements and literature cited. It is a good habit to do this part last in order to avoid confusion and to allow for the writing (and thinking involved) to actually follow the results rather than the results to be bent to prior ideas.

- Write 1-2 sentences that summarize the introduction to the research project. Write one sentence that is a statement of the primary hypothesis or objective. Write 1-2 sentences that summarize the methods used. Write 1-2 sentences that summarize the discussion of the results. Write 1-2 sentences that summarize the conclusions. Put these together as the abstract.

- Produce a title that is descriptive of the research that was done. Avoid cute titles with metaphors unless they provide deeper insight into the research conducted.

- Prepare a list of key words that could be used by other researchers to find this research. Do not repeat words in the title.

- Produce a brief list of acknowledgments, crediting key individuals and organizations who have provided information, data, funding, other support, review of the work, or critical training.

- Check the list of references making sure that all references cited in the text are listed completely in the references and that all references in the references cited are actually cited in the text. 
Appendix 3. A contrived conversation between an ethnobotany research editor and an author from a developing country with limited access to scientific literature.

Editor: "Your research is interesting but the methods are unclear and the results are simply a long table of plants and uses. Could you describe the methods used, citing the literature on methods? Could you discuss the results in light of the cultural context, hypothesis, and finding of other researchers as published in the literature?"

Author: "Thank you. I was trained in country X where we have limited Internet access and few international books or scientific journals. Our library has some literature written during the colonial era, a few older text books, and books written by local authors. We are also able to access research abstracts for papers that are on the Internet. It is difficult for us to find literature that describes current research methods or results."

Editor: "I see. How then did you determine the methods that you used and why is the paper written the way that it is?"

Author: "We conduct our research using the literature that we have as models of how to do the work and then report it. This is the way that we were taught to conduct research and report it. There is a body of similar literature published in our country in local journals and it is hard to understand why this is not acceptable elsewhere."

Editor: "The standards of research are obviously different in different parts of the world. In the developed world, there are expectations that authors will build their research on a global foundation that includes methods, results and theories from the literature that are used to develop hypotheses for current research. The hypotheses are then tested either using proven methods published in the literature or through development of new methods that logically and clearly test the hypothesis."

Author: "The literature that we have available does not include hypotheses in discussions of field biology or anthropology research. Methods are sometimes cited as references but not discussed in enough detail to know how to use the method without access to the literature cited. We have heard that some companies and organizations make literature available free through developing country universities, but this does not appear to be available through my place of work.]"

Editor: "What kinds of methods do you find referenced that you are not able to understand without access to additional journals and books?"

Author: "There are many methods, such as 'Transects', 'Plots', 'Informed Consent', 'Regression Analysis', 'Participant Observation', 'Snow-ball Sampling', 'Walk-in the Woods', and 'Informant Selection', that are discussed as if the reader should know them but remain cryptic to those of us without access to the original papers in which they were described."

Editor: "People and Plants, International has produced a number of documents intended to address the need for field research methods. Some of these, Martin (1995) and Cunningham (2001) have been widely distributed for free or at low cost in many countries and have also been translated into different languages in order to promote ethnobotany research methods."

Author: "I do not have a copy of one of these books, but I have heard of them. Some people in my country have them, but books are very precious and are not easily shared."

Author: "Your journal instructions to authors indicate that authors should use the "Chicago Manual of Style" for preparation of English manuscripts. I cannot find this available on the Internet nor in the libraries in my city. What should I do?"

Editor: "The Chicago Manual of Style is the standard of our journal and many others. Many of the directions in it are based on common sense or traditions that should be apparent from reading English documents produced by Americans. There is a web site (www.press.uchicago.edu/Misc/Chicago/cmosfaq/about.html) that can be used to answer some kinds of questions that the manual addresses. I am afraid that I do not have a good answer to this problem. We do keep in mind that many scholars do not have access to this document and do not count this against them in the review process." 


\section{McClatchey - Improving the Quality of International Ethnobotany Research and Publications}

Editor: "An alternative to publishing in English, if it is not the easiest language for reporting your research, is to write in a more appropriate language. We are able to review and publish research in almost any language. There are some advantages and disadvantages to this process that should be considered as have been discussed by McClatchey and Winter (2005)."

Editor: "So, what can I do to help you and others in your situation to access research methods? This is particularly hard since many of the publications needed are accessible to me through my University library, but are protected by copyright laws that prohibit me from simply sending copies of the publications to you."

Author: "Provide clear, step by step directions on how to use each method and how to interpret the results. If this includes a discussion of the when and why to use the methods, this will help us to select the one that is most appropriate. A discussion of how well each method works with a literature review of its use will also help us understand the global context of the method as it addresses hypotheses and is used to generate new hypotheses and theories." 
